\title{
A SIMULATION FRAMEWORK FOR REAL-TIME MANAGEMENT AND CONTROL OF INVENTORY ROUTING DECISIONS
}

\author{
Shrikant Jarugumilli \\ Scott E. Grasman \\ Sreeram Ramakrishnan \\ Dept. of Engineering Management and Systems Engineering \\ University of Missouri - Rolla, \\ Rolla, MO 65409, U.S.A.
}

\begin{abstract}
We consider a logistics network where a single warehouse distributes a single item to multiple retailers. Retailers in the network participate in a Vendor Managed Inventory (VMI) program with the warehouse, where the warehouse is responsible for tracking and replenishing the inventory at various retailer locations. The information update occurs every time a vehicle reaches a location and the decision on the delivery quantity and the next location to visit is made. For a small increase of locations in the network, the state space for the solution increases exponentially, making this problem NP-hard. Thus, we propose a solution methodology where in the size of the state space is reduced at each stage. In this work, we use simulation to develop the framework for the real-time control and management of inventory and routing decisions, given this scenario.
\end{abstract}

\section{INTRODUCTION}

In an efficient logistic network the primary objective is to deliver the right product, in the right quantity, at the right time, while keeping the overall cost at a minimum. A typical logistics network consists of multiple factories, distribution centers, wholesalers, retail outlets, and customers, and requires movement of material/ inventory in the network to be efficient and economical by making optimal routing and inventory decisions.

Advances in technology have forced many companies to change their business models, and many companies participate in VMI initiatives with their suppliers. The tangible benefits of successful VMI implementation include improved forecasting due to increased visibility across the supply chain, reduced inventory levels with higher inventory turns, and reduced costs (Kleywegt, Nori, and Savelbergh 2002; Lee and Whang 1998). The retailers also gain remarkable efficiencies by reducing errors and stockouts, while increasing customer satisfaction. Hence, it becomes essential for a supplier to follow an integrated approach for making optimal routing and inventory decisions.

Inventory routing problems (IRP), integrate the management and control of inventory and vehicle routing in a logistics network. The complexity of IRP lies in the fact that there could be several alternative routes among the various retailer locations in the network and a varying quantity of inventory to be allocated at each location. Intuitively, it is ideal to satisfy the demand at each location when requested, however, real life situations are constrained by the vehicle capacity and fluctuating demand patterns at other locations.

We refer the readers to the work of Kleywegt, Nori, and Savelbergh (2002) and Kleywegt, Nori, and Savelbergh (2004), where the inventory routing problem is formulated as a Markov decision process (MDP). Adelman (2003 a) and Adelman (2003 b) also formulate the IRP control problem as a MDP, approximating future costs of current actions using optimal dual prices from a linear programming model. Also, the readers are referred to the works of Campbell and Savelsbergh (2004 a), Campbell and Savelsbergh (2004 b) and Campbell and Savelsbergh (2004 c) for the various approaches taken to formulate and solve the IRP.

In this paper, we consider a situation where one warehouse replenishes inventory at multiple retailers using a single vehicle. The vehicle is scheduled to visit all the locations where the inventory level is below the reorder point at any time during the visit. The demand pattern at each location is stochastic and stationary; hence causing a variation in the actual and predicted inventory at some or all locations. During the visit of the vehicle to the planned locations there may be an addition of other locations where the inventory is below the reorder point. To accommodate every new location that enters a route or to cater to the fluctuating demands at the locations on the current route delivery quantity needs to be varied and/or the vehicle is rerouted. After visiting each location the decision on which 
location to visit next and how much to deliver at the present location are taken. Finally, the vehicle returns back to the warehouse when the inventory on the vehicle is exhausted or so low that it is not desirable to service any more locations on the route.

We formulate the sequential decision problem as a stochastic dynamic program considering the sequential decision making. The solution space for this type of the problems is extremely large making the problem NP-hard; however, we modify the $A^{*}$ algorithm in order to find good heuristic solutions. We develop a simulation framework to validate the heuristic performance for the real-time management and control of inventory routing decisions.

The remainder of the paper is organized as follows. Section 2 presents problem definition, followed by section 3 , which presents the modified $\mathrm{A} *$ algorithm. The numerical analysis is presented in section 4, results and discussions in section 5, followed by the conclusion in Section 6.

\section{PROBLEM DEFINITION}

In this paper, we focus on the relationship between a single warehouse and multiple retailers. The retailers participate in a Vendor Managed Inventory program, where the wholesaler is responsible for tracking and replenishing the inventory in a timely manner. We consider the demand at the retailer locations to be stochastic and stationary.

\subsection{Notation}

Prior to describing the model formulation, a list notation used throughout the paper is provided below:

Network representation:

$\mathrm{k} \in \mathrm{K} \quad \mathrm{k}=0$ represents the warehouse $\mathrm{k}=1,2,3 \ldots \mathrm{K}$ represent the retailer locations

$\mathrm{c}_{\mathrm{ij}}=$ cost of operating between locations $\mathrm{i}$ and $\mathrm{j}$

$\mathrm{d}_{\mathrm{ij}}=$ distance between locations $\mathrm{i}$ and $\mathrm{j}$

$\mathrm{t}_{\mathrm{ij}}=$ travel time from locations $\mathrm{i}$ to $\mathrm{j}$

Inventory modeling parameters for location $k$

$\mathrm{a}_{\mathrm{k}} \quad=$ setup cost for each order

$\mathrm{p}_{\mathrm{k}} \quad=$ shortage cost per unit

$\mathrm{h}_{\mathrm{k}} \quad=$ holding cost of inventory

$\mathrm{w}_{\mathrm{k}} \quad=$ unit cost

$\Phi_{\mathrm{k}}{ }^{\lambda}(\mathrm{d})=$ normal distribution of demand during time pe$\operatorname{riod} \lambda$

$\theta_{\mathrm{k}} \quad=$ expected demand during lead time

$\sigma_{\mathrm{k}} \quad=$ standard deviation of demand during leadtime

$(\mathrm{R}, \mathrm{s}, \mathrm{S})_{\mathrm{k}}=$ the periodic review model where R-review interval, s-reorder point, S-order upto quantity

\section{Cost Functions}

$\mathrm{F}(\mathrm{t})$ Total cost of operation from the warehouse through node $\mathrm{t}$
$G(t)$ Cost of reaching the node $t$ from the warehouse

$\mathrm{H}(\mathrm{t})$ Cost of reaching the warehouse from node $t$

$F^{t}(j)$ Total cost of operation from the warehouse through node $t$ in the modified $A^{*}$ algorithm

$G^{t}(j)$ Cost of reaching the node $t$ from the warehouse in the modified A* algorithm

$\mathrm{H}^{t}(\mathrm{j})$ Cost of reaching the warehouse from node $t$ in the modified A* algorithm

State notation

$\mathrm{T}=$ node visitation counter

(t) $=$ index of the $\mathrm{t}^{\text {th }}$ visited node

$\mathrm{I}_{\mathrm{k}}{ }^{\mathrm{t}}=$ actual inventory at location $\mathrm{k}$ upon arrival at the $\mathrm{t}^{\text {th }}$ node

$\tilde{\mathrm{I}}_{\mathrm{k}}{ }^{\mathrm{t}}=$ predicted inventory at location $\mathrm{k}$ upon arrival at the $t^{\text {th }}$ node

$Q^{t}=$ inventory on vehicle upon arrival at the $t^{\text {th }}$ node

Decision variables

$D_{k}{ }^{t}=$ inventory delivered to location $\mathrm{k}$ while at the $\mathrm{t}^{\text {th }}$ node

$\mathrm{x}_{\mathrm{ij}}{ }^{\mathrm{t}}=$ binary variable associated with selection of the route

$$
\left\{\begin{array}{l}
0, \text { if }(t)=i \text { and }(t+1)=j \\
1, \text { otherwise }
\end{array}\right.
$$

\subsection{Problem Description}

In this problem, we consider a single product distributed from a single distribution center to $\mathrm{k}$ wholesaler locations. Locations are indexed $\mathrm{k}=0,1,2, \ldots, \mathrm{K}$, where $\mathrm{k}=0$ represents the distribution facility. Inventory on a single distribution vehicle, while at any location $t$, is expressed as $Q^{t}$ and changes as the vehicle delivers to various locations according to:

$$
Q^{t+1}=Q^{t}-D_{(t)}^{t},
$$

where $\mathrm{D}_{(\mathrm{t})}{ }^{\mathrm{t}}$ represents the inventory delivered at the location while at node $t$ and $(t)$ is the index of the interval between each decision epoch. Upon reaching zero inventory, or a level where it might not be desirable to service any other additional locations, the vehicle returns to the distribution center prior to starting a new tour; any unvisited locations are unsatisfied and may incur penalties. The inventory routing processing is described in Figure 2.1.

We choose a periodic review model, $(\mathrm{R}, \mathrm{s}, \mathrm{S})_{\mathrm{k}}$ to determine inventory control parameters for each location as it is consistent with the stochastic nature associated with the inventory routing problem. A power approximation, proposed by Ehrhardt (1979) and Ehrhardt and Mosier (1984) is used to determine optimal values of the reorder point, $\mathrm{s}_{\mathrm{k}}$, 
the order up to level, $S_{k}$ as shown in equation (2) and (3). Each time the vehicle reaches a location the inventory at all the locations is reviewed, hence the review of inventory occurs each time a location is visited. The reorder point is set as a threshold value to assess the criticality of servicing a location.

$$
\begin{gathered}
S_{k}=1.3\left(\theta_{k}\right)^{.494}\left(\frac{a_{k}}{h_{k}}\right)^{0.506}\left(1+\frac{\sigma_{k}^{2}}{\theta_{k}^{2}}\right)^{0.116} \\
S_{k}=0.973 \theta_{k}+\sigma_{k}\left(\frac{0.183}{z_{k}}+1.063-2.192 z_{k}\right)
\end{gathered}
$$

Where $z_{k}=\sqrt{S_{k} \frac{w_{k}}{\sigma_{k} b_{k}}}$.

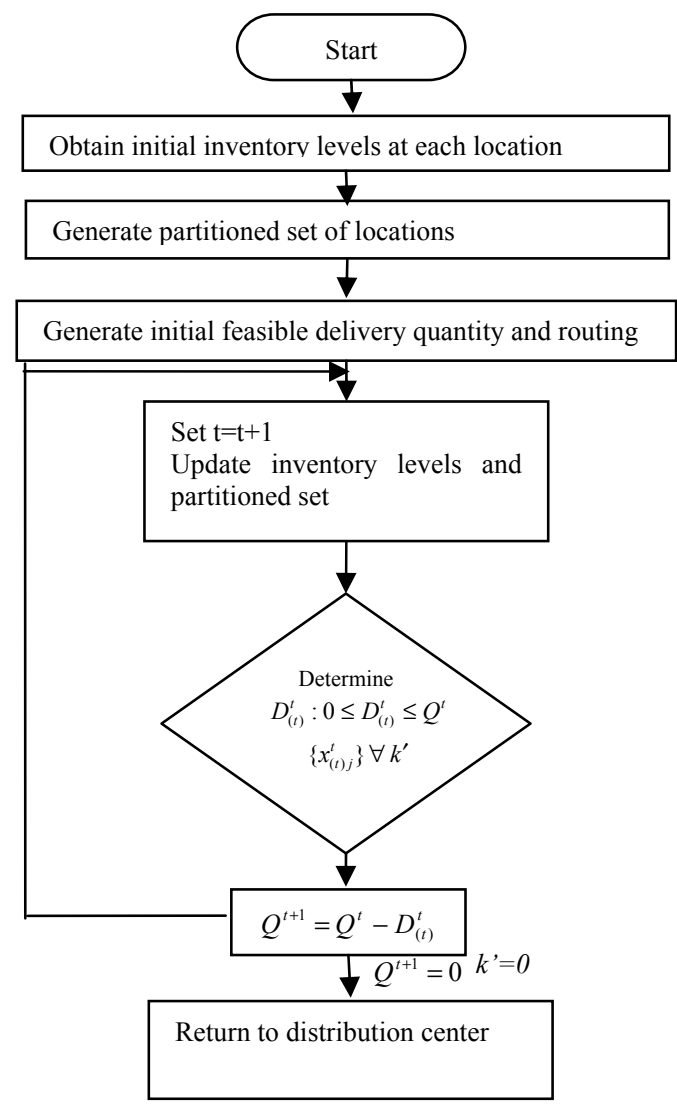

Figure 2.1. Inventory/Routing Processing Flowchart (Adapted from Jarugumilli and Grasman (2006))

The inventory at each location $\mathrm{k}$ is reviewed each period. If the inventory level falls below $s_{k}$ an order is placed at the distribution center in order to replenish the inventory to $\mathrm{S}_{\mathrm{k}}$. Within this framework, there are two decision epochs:

1. generation of initial feasible (optimal) delivery quantities and routing, and
2. determination of feasible delivery quantity at location $\mathrm{k}$ while at the $\mathrm{t}^{\text {th }}$ node $\mathrm{D}_{(\mathrm{t})}{ }^{\mathrm{t}}$ and the decision on the next node to be visited while at the $t^{\text {th }}$ node $\left\{x_{(t) j}{ }^{t}\right\}$ based on updated information.

The first decision may be made using existing vehicle and inventory routing literature. The second decision deals with real-time control and is presented in Section 3.

The cost of operations in the entire network is the sum of inventory holding and shortage costs and transportation costs. Generally the holding costs are defined as the cost of holding the inventory until it is consumed. The shortage costs are defined as the losses that the wholesaler incurs due to shortage of an item and are generally higher than the holding costs. The transportation costs are defined as the costs incurred to travel between two or more locations; this is directly proportional to the distance between the locations.

We formulate the problem as a stochastic dynamic program. This approach is adopted considering the sequential decision making. The present state of the system determines the allowable actions and the possible states to which the system can transition. The present formulation involves the following components.

The current state of the system at any instant is defined as: the current and the predicted inventory at all the locations in the network, the current location of the vehicle in the network and the amount of inventory on the vehicle. Depending on the current state the decisions are made and the system transitions into the future state. The action space for any given state is the set of all decisions that can be taken without violating the vehicle and retailer capacity constraints and the routing constraints. The constraint that the retailer capacity is not violated is expressed as $\mathrm{I}_{\mathrm{k}}{ }^{\mathrm{t}}+$ $\mathrm{D}_{(\mathrm{t})}{ }^{\mathrm{t}}<\mathrm{S}_{\mathrm{k}}$. The constraints for the vehicle capacity can be expressed as $Q^{t} \geq Q^{t+1}$, where $t=1,2,3 \ldots T$.

The objective is to minimize the system wide costs considering the various states without violating the capacity and routing constraints. We set up the problem as a recursive function, where the total cost is the sum of costs incurred at each state transition; thus:

$$
\begin{gathered}
f_{t}\left(k, I_{k}^{t}, \widetilde{I}_{k}^{t+1}, Q^{t}\right)=\min _{\substack{j \in k^{\prime} \\
0 \leq D_{(t)}^{t} \leq Q^{t}}}\left\{c_{k j}+Y_{k}\left(D_{(t)}^{t}\right)+f_{t+1}\left(j, I_{j}^{t+1}, \widetilde{I}_{j}^{t+1}, Q^{t+1}\right)\right\} \\
Y_{k}\left(D_{(t)}^{t}\right)=h_{k} \sum_{d=0}^{D_{(t)}^{t}+I_{k}^{t}}\left(\left(D_{(t)}^{t}+I_{k}^{t}\right)-d\right) \Phi^{\lambda}(d)+b_{k} \sum_{d=D_{(t)}^{t}+I_{k}^{t}}^{\infty}\left(d-\left(D_{(t)}^{t}+I_{k}^{t}\right)\right) \Phi^{\lambda}(d)
\end{gathered}
$$

Equation (4), adopted from Jarugumilli and Grasman (2006), provides the inventory control policy cost component of the dynamic program, including inventory carrying cost and shortage cost associated with the delivery quantity. The policy cost is calculated using equation (5). 


\section{A * ALGORITHM}

In this section, we describe the solution methodology for the stochastic dynamic program. In the first phase we determine the initial inventory control parameters using an $(\mathrm{R}, \mathrm{s}, \mathrm{S})_{\mathrm{k}}$ model as discussed earlier in Section 2. The second phase involves the use of $A^{*}$ algorithm to dynamically determine the deliver quantities and routing each location.

In this section, we describe the $\mathrm{A}^{*}$ for the stochastic dynamic program, described in Equation 4. Solving this class of problem is complex because of the large number of solution states to which the system can transit into. The complexity of the problem is reduced to the linear form and a variant of the $\mathrm{A}^{*}$ algorithm is proposed to dynamically determine the deliver quantities and routing.

The $A^{*}$ algorithm is a graph search algorithm that finds a path from a given initial node to a given goal node. The algorithm calculates the cost of travel from an initial node to the final node as the sum the cost of reaching the intermediate node from the initial node and the estimated cost of reaching the final node from the intermediate node. Hence, the cost, $\mathrm{F}(\mathrm{t})$, is expressed as:

$$
F(t)=G(t)+H(t)
$$

Where $G(t)$ the cost of traveling from the initial node to the intermediate node $\mathrm{t}, \mathrm{H}(\mathrm{t})$ is the estimated cost of traveling from the node $t$ to the final node.

From the Equation 6, it is evident that the algorithm can be effectively used for sequential decision making problems like the dynamic program presented in the Equation 4. G(t) calculates the cost of travel to the present location $\mathrm{t}$, and $\mathrm{H}(\mathrm{t})$ calculates the cost of travel for the forward recursion, i.e., from the present location $t$ to the final destination.

The problem formulation in Equation (4), considers minimizing the total cost in the network, which is comprised of the travel cost and inventory cost in the network. The cost components $G(j)$ and $H(j)$ in the original form used for the $\mathrm{A}^{*}$ algorithm consider only the transportation cost. In order to account for the inventory cost we slightly modify $G(t)$ and $H(t)$. Now, $G^{t}(j)$ is defined as the sum of the cost of traveling from the initial node to the present node $t$ and the cost of inventory for all the locations covered on route from the initial node to the present node t. Similarly, the modified $\mathrm{H}^{\mathrm{t}}(\mathrm{j})$ is the sum of the estimated cost of travel from the present node $t$ to the final node and the estimated cost of inventory for all locations to be covered on route to the final node (which in our case is the warehouse).

At any given point in time $G^{t}(j)$ can be determined as the sum of the costs incurred to reach the current location, but $\mathrm{H}^{\mathrm{t}}(\mathrm{j})$ is sum of the cost of servicing the remaining locations on the partitioned set, which is subject to change in the future based on the elements of the partitioned set. We calculate the costs $F^{t}(j)$ of getting to both the locations based on Equations 7 and 8.

$$
\begin{gathered}
\mathrm{G}^{\mathrm{t}}(\mathrm{j})=\min _{j}\left\{G^{t-1}(j)+c(t-1) t+Y\left(D_{(t)}^{t}\right)\right\} \\
\mathrm{H}^{\mathrm{t}}(\mathrm{j})=\min _{j}\left\{\sum_{k=t}^{T-1}\left(c_{(t)(t+1)}\right)+c_{(T)(0)}+\sum_{k=t}^{T-1} Y\left(D_{(t)}^{t}\right)\right\}
\end{gathered}
$$

Initially, all the locations are in the closed list. While the vehicle is at a location $t$, the updated inventory information from all the locations in the network is obtained and the partitioned set is updated with all locations where the inventory $\mathrm{I}_{\mathrm{k}}<\mathrm{s}_{\mathrm{k}}$. All locations in the partitioned set are added to the open list. $F^{t}(j)$ is then calculated for all the locations and the one with the minimum $F^{t}(j)$ is visited. On reaching the location, the inventory is again updated. If the sum of requirements at all the locations is less than the truck capacity, then the pre-allocated inventory is delivered at the current location. If the sum of inventory requirements at the locations is greater than the inventory on the truck, adjustments may be made to the inventory allocation and the routing decisions. There are several ways of allocating the inventory and visiting future locations in the network, causing an increase in the number of states to which the system can transition. To reduce the size of state space, we put a restriction on the adjustment that can be made to the inventory allocation (Jarugumilli and Grasman 2005) at a particular location. At each location, there is a possibility of adjusting the delivery quantities to three levels:

1. Deliver the originally allocated delivery quantity,

2. Deliver the difference of the original delivery quantity and the sum of requirements in the future nodes, and,

3. Deliver the updated delivery quantity.

The inventory to be delivered at a location is determined by calculating the total $\operatorname{cost} F^{t}(j)$. We calculate $G^{t}(j)$ considering the above mentioned three levels of inventory for the current location. Next we calculate the cost of servicing the future locations $\mathrm{H}^{\mathrm{t}}(\mathrm{j})$, based on the various levels of inventory on the truck resulting from the amount delivered at the current location and the possible route change. The priority is given to the locations where the cost of servicing is the highest if not serviced, i.e. to locations with a low $\mathrm{F}^{\mathrm{t}}(\mathrm{j})$. After delivering the inventory at the current location, the current location is added to the closed list and decision on the location to be visited next is taken based on $F^{t}(j)$.

Intuitively, if a location is not serviced at the right time, this will result in a higher value of $\mathrm{H}^{\mathrm{t}}(\mathrm{j})$, resulting in a higher value of $F^{t}(j)$, forcing the vehicle to visit the particular location to keep the costs low. Hence, the minimum $\operatorname{cost} F^{t}(j)$ is chosen and the corresponding inventory level to be delivered at the current location and the next location to be visited is chosen. At every location that is visited 
these calculations are made until the inventory on the truck reduces to a level where visiting a location is no longer desirable or all the elements in the partitioned set are visited. The algorithm is tabulated in Table 3.1.

Table 3.1: Modified A* Algorithm

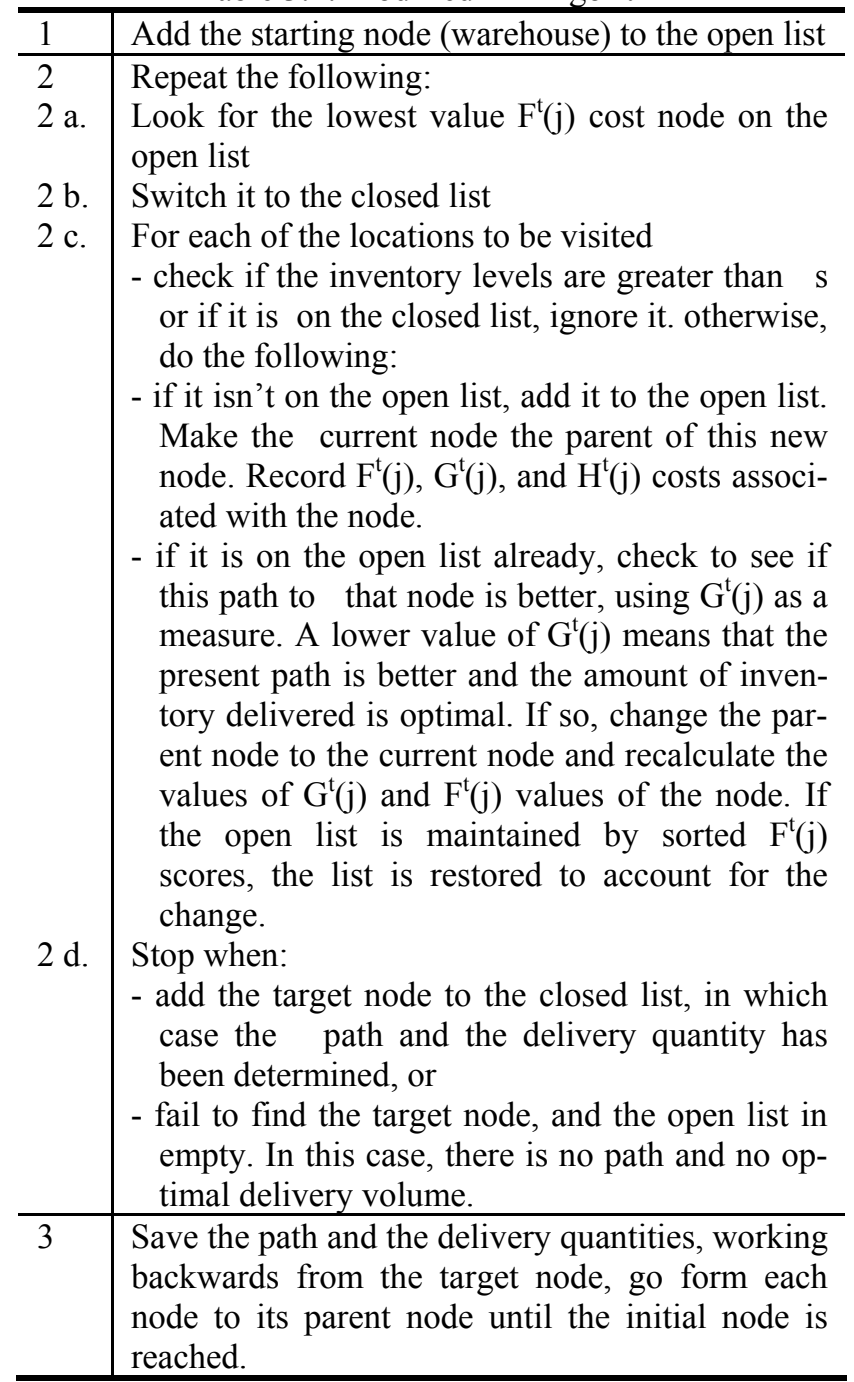

\section{NUMERICAL ANALYSIS}

In this section, we describe the design of experiments used for validation of the model. We present the numerical analysis to test a series of hypotheses related to the effect of factor levels on the cost of operations in the network.

\subsection{Objectives of the numerical analysis}

The main objectives of the numerical analysis are to study the following:

1. Perform statistical analysis of the cost savings in the network,
2. Analyze the effect of the demand distribution, and inventory control costs on the overall cost of operations, and,

3. Discuss the scalability of the heuristic to handle problems of larger size.

\subsection{Design of Experiments}

For the experimental design, three important design factors were identified: the demand pattern, the ratio of the shortage cost to the holding cost, and the transportation cost. The demand at each of the retailer locations is a common source of randomness in the system, and based on the demand, the inventory control parameters $\left(\mathrm{s}_{\mathrm{k}}, \mathrm{S}_{\mathrm{k}}\right)$ for each of the locations are calculated. The inventory levels at each location act as a trigger for adjusting the delivery quantities and the routes in order to minimize the total inventory and transportation costs. Also, the shortage costs and the transportation cost have a significant effect on the cost savings. The three factors and respective levels are tabulated in Table 4.1.

Table 4.1. Experimental Design

\begin{tabular}{|l|l|l|l|l|l|l|}
\hline Factor & Name & Notation & \multicolumn{3}{|l|}{ Levels } \\
\cline { 4 - 8 } & & & 1 & 2 & 3 & 4 \\
\hline 1 & $\begin{array}{l}\text { Demand } \\
\text { Normal }\end{array}$ & $\theta_{\mathrm{k}} / \sigma_{\mathrm{k}}$ & 5 & 10 & 15 & \\
\cline { 2 - 7 } & Uniform & $\mathrm{a} / \mathrm{b}$ & .5 & .33 & .25 & \\
\hline 2 & $\begin{array}{l}\text { Shortage cost vs. } \\
\text { Holding cost }\end{array}$ & $\mathrm{p}_{\mathrm{k}} / \mathrm{h}_{\mathrm{k}}$ & 5 & 10 & 15 & 20 \\
\hline 3 & $\begin{array}{l}\text { Transportation } \\
\text { Cost }\end{array}$ & $\Gamma$ & 5 & 10 & 15 & 20 \\
\hline
\end{tabular}

The full-factorial design for these factors and levels requires a $6 \times 4 \times 4$, i.e. a 96 trial experiment. Each trial consists of ten replications; of 450 simulated periods.

\subsubsection{Data Input}

The simulation model uses the following input data:

1. Demand distribution at each retailer location.

2. Inventory control parameters for each retailer location based on the periodic review model.

3. Inventory holding and shortage cost at each location.

4. Capacity of the delivery vehicle.

5. Cost of traveling from one location to the other.

6. Inventory levels at each of the retailer locations.

The demand at each of the retailer locations is the common source of randomness in the system. In this model, the demand is considered to be stationary and stochastic, following a normal distribution. Based on the de- 
mand, the inventory control parameters for each of the locations are calculated. Inventory levels at each of the locations vary with the demand each period, making it a random function. The inventory levels at each location acts as a trigger based on which the delivery quantities and the routes are adjusted, so that the total inventory and transportation costs are minimum.

\subsubsection{Model Verification and Validation}

The initial verification of the model included the following steps adopted from Law and Kelton (2003):

1. the model was programmed and debugged in steps

2. to test each program path the debugger was used extensively.

3. model output results were checked for reasonableness

4. model summary statistics for the values generated from the input probability distributions were compared to that with a network with fixed route and delivery quantity.

The process of validation of this scenario is a difficult process. We have compared our assumptions and constraints with the existing literature for logical correctness and accuracy. Different data sets for the same randomness were tested for homogeneity and merged only if appropriate. We tested all the probability distribution for the correctness.

\subsection{Output Analysis}

In this section, we present the output of the numerical analysis mentioned in the previous section. We will present and test hypotheses dealing with the impact of individual design factors on the experiments and the interactions among various factors, based on the results the results of the 96 trials. The mean percentage, savings, is the response variable, is the mean value of the percentage savings for 10 replications of the same trial. The 96 trials were conducted for three values of $\theta$ in case of the normal distribution and three values of a in case of the uniform distribution.

\subsubsection{Main Effects}

In this subsection, we test the hypotheses to check the significance of the levels of the individual factors. The hypotheses testing are done for the factors: demand, shortage cost and the transportation cost. Figure 4.1-4.2 show the main effect plots at different values of mean and a for the normal and uniform distribution respectively.

\section{Hypothesis 1:}

$\mathrm{H}_{0}$ : Levels of factor for 'Demand' does not change the mean percentage saving.
$\mathrm{H}_{\mathrm{a}}$ : Levels of factor for 'Demand' changes the mean percentage saving.

$\mathrm{H}_{0}: \mu_{1}=\mu_{2}=\mu_{3}$;

$\mathrm{H}_{\mathrm{a}}: \mu_{1} \neq \mu_{2 ; \mu 2} \neq \mu_{3}$; Test for the alternate hypotheses was carried out separately.

The results of the paired t-test conducted for the three levels of demand for normal and uniform distribution. The $\mathrm{p}-$ values for all the factors $<0.05, \mathrm{H}_{0}$ is rejected and $\mathrm{H}_{\mathrm{a}}$ is accepted.

\section{Hypothesis 2:}

$\mathrm{H}_{0}$ : Levels of factor 'Shortage Costs' does not change the mean percentage saving.

$\mathrm{H}_{\mathrm{a}}$ : Levels of factor 'Shortage Costs' changes the mean percentage saving.

$\mathrm{H}_{0}: \mu_{1}=\mu_{2}=\mu_{3}=\mu_{4}$;

$\mathrm{H}_{\mathrm{a}}: \mu_{1} \neq \mu_{2}$

$\mu_{2} \neq \mu_{3}$

$\mu_{3} \neq \mu_{4}$; Test for the alternate hypotheses was carried out separately.

The results of the paired t-test conducted for factors shortage cost for normal and uniform distribution. The p-values for all the factors $<0.05, \mathrm{H}_{0}$ is rejected and $\mathrm{H}_{\mathrm{a}}$ is accepted.

Hypothesis 3:

$\mathrm{H}_{0}$ : Levels of factor 'Transportation Costs' does not change the mean percentage saving.

$\mathrm{H}_{\mathrm{a}}$ : Levels of factor 'Transportation Costs' changes the mean percentage saving.

$\mathrm{H}_{0}: \mu_{1}=\mu_{2}=\mu_{3}=\mu_{4}$

$\mathrm{H}_{\mathrm{a}}: \mu_{1} \neq \mu_{2}$

$\mu_{2} \neq \mu_{3}$

$\mu_{3} \neq \mu_{4}$; Test for the alternate hypotheses was carried out separately.

The results of the paired t-test conducted for transportation cost for normal and uniform distribution. The $p$-values for all the factors $<0.05, \mathrm{H}_{0}$ is rejected and $\mathrm{H}_{\mathrm{a}}$ is accepted.

\subsubsection{Two Factor Interaction}

In this subsection, we test the hypotheses to check the significance of the levels of the interaction between two factors. The hypotheses testing are done for the interaction between the factors: demand, shortage cost and the transportation cost. Figures $4.3-4.4$ show the interaction plots between the factors.

Hypothesis 4:

$\mathrm{H}_{0}$ : Change in the levels of factors Demand and Shortage cost does not alter the mean percentage saving.

$\mathrm{H}_{\mathrm{a}}$ : Change in the levels of factors Demand and Shortage cost alters the mean percentage saving. 
The results of the two-way ANOVA test conducted for factors demand (for normal and uniform distribution) and shortage cost. The $\mathrm{p}$ values for all the factors $>0.05, \mathrm{H}_{0}$ is not rejected for the normal demand. The $\mathrm{p}$ values for all the factors $<0.05, \mathrm{H}_{0}$ is rejected and $\mathrm{H}_{\mathrm{a}}$ is accepted for uniform demand.

Hypothesis 5:

$\mathrm{H}_{0}$ : Change in the levels of factors Demand and transportation cost does not alter the mean percentage saving.

$\mathrm{H}_{\mathrm{a}}$ : Change in the levels of factors Demand and transportation cost alters the mean percentage saving.

The two-way ANOVA test conducted for factors Demand and Transportation Cost. The $\mathrm{p}$ values for all the factors $>$ $0.05, \mathrm{H}_{0}$ is not rejected.

\section{Hypothesis 6:}

$\mathrm{H}_{0}$ : Change in the levels of factors shortage cost and transportation cost does not alter the mean percentage saving.

$\mathrm{H}_{\mathrm{a}}$ : Change in the levels of factors shortage cost and transportation cost alters the mean percentage saving.

The two-way ANOVA test conducted for factors shortage cost and transportation cost. The $\mathrm{p}$ values for all the factors $>0.05, \mathrm{H}_{0}$ is not rejected.

\section{DISCUSSION OF RESULTS}

The analysis performed on the data sets with normal and uniform distributions can be summarized as:

1. The shortage cost is a significant factor in the system, and the locations with higher shortage costs must be on the priority of service.

2. The demand is a significant factor in the system, variability increases, the mean percentage saving reduces.

3. The transportation cost is a significant factor in the system.

4. For change in the levels of factors of demand and the shortage costs, there is a change in the mean percentage savings only for cases where the variances are high. In this study, the data set for normal demand does not reflect this fact mainly due to the lesser variation in the demand pattern. This concept is well reflected in the uniform distribution data set.

5. In this study, there is no interaction observed between the transportation cost and the demand.

6. Again, there was interaction observed between the transportation cost and the shortage cost.

The results show that there can be some substantial savings achieved by the implementation of the connective technology in the logistics network, which will enable the real-time information exchange between the vehicle and the various locations in the network. The use of connective technology, will aid in the real-time decision making on dynamically determining the delivery quantities and rerouting of the vehicle.

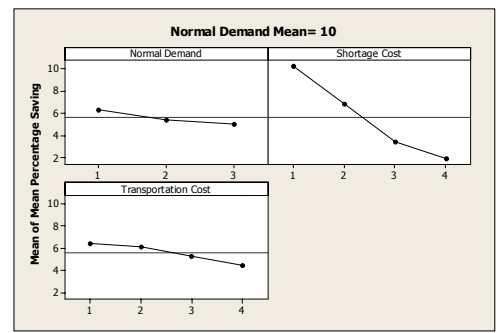

Figure 4.1 Main Effect Plots for Normal Demand, Mean $=10$

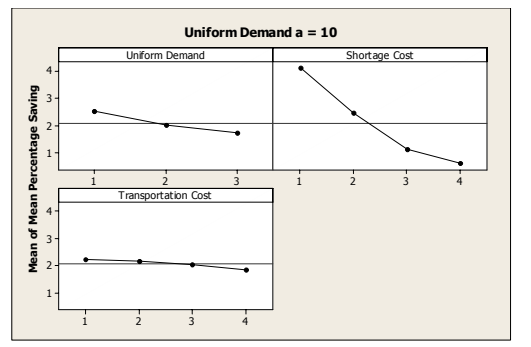

Figure 4.2 Main Effect Plots for Uniform Demand, $\mathrm{a}=10$

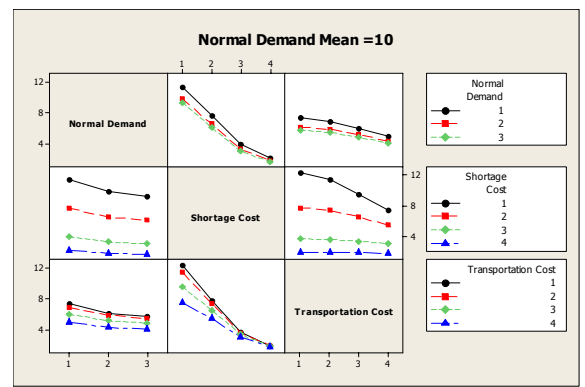

Figure 4.3 Interaction Plots for Normal Demand, Mean=10

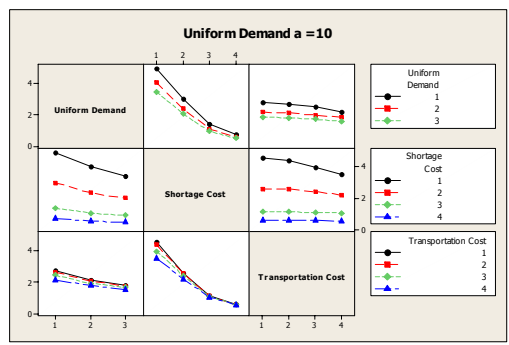

Figure 4.4 Interaction Plots for Uniform Demand, a =10

\section{CONCLUSION}

In this paper, a methodology for dynamic control of a inventory and routing decisions in a logistics network is pre- 
sented. Using a vendor managed inventory approach; we compare the cost of operations in networks enabled by the connective technologies with networks not implementing such technology. The problem has been set up as a stochastic dynamic program. $\mathrm{A}^{*}$ algorithm is used to solve this problem and to bring out the cost benefit associated with the dynamic control of inventory and routing decisions. We have presented an extensive numerical analysis to test the efficiency of the methodology.

This model should be considered along with the set up cost associated with ordering each time an order is placed. The model may be extended to more complex systems, such as systems with multiple vehicles or multiple distribution centers. In these situations, the inventory routing problem may require partitioning algorithms, in order to deal with nature of the vehicle routing problem. Other extensions include current advances in VRP and IRP, such as anticipatory route selection probabilistic routing, or delivery windows. Setting of dynamic threshold values for each of the locations will help in more optimized operations in the logistics network. This model can be extended to include use of multiple vehicles in the network, the distribution of multiple products and considering the distribution of perishable inventory.

\section{REFERENCES}

Adelman, D. 2003. Price-directed replenishment of subsets: methodology and its application to inventory routing. Manufacturing Service Operation Management 5(4): 348-371

Adelman, D. 2003. A price-directed approach to stochastic inventory/routing. Operations Research 52(4): 499-514

Campbell, A.M., and M. W. P. Savelbergh. 2004. A decomposition approach for the inventory- routing problem. Transportation Science 38(4): 488-502

Campbell, A.M., and M. W. P. Savelbergh. 2004. Delivery volume optimization. Transportation Science 38(2): 210-223

Campbell, A.M., and M. W. P. Savelbergh. 2004. Efficient insertion heuristics for vehicle routing and scheduling problems. Transportation Science 38(3): 369-378

Ehrhardt, R. 1979. The power approximation for computing $(\mathrm{s}, \mathrm{S})$ inventory policies. Management Science 25(8): 777-786

Ehrhardt, R., and C. Mosier. 1984. A revision of the power approximation for computing $(\mathrm{s}, \mathrm{S})$ policies. Management Science 30(5): 618-622

Jarugumilli, S., and S. E. Grasman. 2005. An integrated inventory and routing decision framework in onewarehouse multi- retailer systems using RFID technology, In Proceedings of the International Conference on Operations Research Applications in Infrastructure Development
Jarugumilli, S., and S. E. Grasman. 2006. RFID-enabled inventory routing problems. Int. J. Manufacturing Technology and Management. To Appear in the Special Issue on Connective Technologies and Their Impact on Manufacturing and Logistics

Kleywegt, A., V. Nori, and M. W. P. Savelbergh. 2002. The stochastic inventory routing problem with direct deliveries. Transportation Science 36(1): 94-118

Kleywegt, A., V. Nori, and M. W. P. Savelbergh. 2004. Dynamic programming approximations for a stochastic inventory routing problem. Transportation Science 38(1): $42-70$

Law, A. M., and D. W. Kelton. 2003. Simulation Modeling and Analysis. $3^{\text {rd }}$ Edition, New York, McGraw-Hill

Lee, H. L., and S. Whang. 1998. Information sharing in a supply chain. Research paper No. 1549, Research Papers Series, Graduate School of Business, Stanford University

\section{AUTHOR BIOGRAPHIES}

SHRIKANT JARUGUMILLI is a graduate student currently working as a graduate research assistant in the Department of Engineering Management and Systems Engineering at University of Missouri, Rolla. He has a B.E. (Industrial Engineering and Management) from Visvesvaraya Technological University, India. His research interests are inventory routing problems and dynamic programming. $\mathrm{He}$ is a member of IIE and INFORMS. His e-mail address is $<$ sjarugumilli@gmail. com $>$.

SCOTT GRASMAN is an Assistant Professor of Engineering Management and Systems Engineering at the University of Missouri-Rolla. Dr. Grasman received his B.S.E., M.S.E., and Ph.D. degrees in Industrial and Operations Engineering from the University of Michigan. His primary research interests relate to the application of quantitative models to manufacturing and service systems, focusing on the design and development of supply chain and logistics. He has received research funding from, among others, National Science Foundation, US Department of State, and SAP America. His e-mail address is <grasmans@umr. edu>.

SREERAM RAMAKRISHNAN is an assistant professor of Engineering Management and Systems Engineering in the University of Missouri- Rolla. He has a Ph.D. in Industrial Engineering from Pennsylvania State University, a M.S. (Industrial Engineering) from Binghamton University, and a B.Tech. (Mechanical) degree from University of Kerala, India. His research interests include distributed systems modeling, simulation-based control, and modeling control systems. He is a member of IIE and ASEE. His email address is $<$ sreeram@umr. edu $>$. 